SUBJECT AREAS:

NANOPHOTONICS AND

PLASMONICS

METAMATERIALS

Received

4 December 2013

Accepted

17 January 2014

Published

4 February 2014

Correspondence and requests for materials should be addressed to

T.C. (caotun1806@ dlut.edu.cn)

\title{
Broadband Polarization-Independent Perfect Absorber Using a Phase-Change Metamaterial at Visible Frequencies
}

\author{
Tun Cao', Chen-wei Wei', Robert E. Simpson³, Lei Zhang' \& Martin J. Cryan²
}

\begin{abstract}
'Department of Biomedical Engineering, Faculty of Electronic information and Electrical Engineering, Dalian University of Technology, Dalian, China(P.R.C), ${ }^{2}$ Department of Electrical and Electronic Engineering, University of Bristol, Bristol, UK, ${ }^{3}$ Singapore University of Technology and Design (SUTD), 20 Dover Drive, Singapore.
\end{abstract}

We report a broadband polarization-independent perfect absorber with wide-angle near unity absorbance in the visible regime. Our structure is composed of an array of thin Au squares separated from a continuous $\mathrm{Au}$ film by a phase change material $\left(\mathrm{Ge}_{2} \mathrm{Sb}_{2} \mathrm{Te}_{5}\right)$ layer. It shows that the near perfect absorbance is flat and broad over a wide-angle incidence up to $80^{\circ}$ for either transverse electric or magnetic polarization due to a high imaginary part of the dielectric permittivity of $\mathrm{Ge}_{2} \mathrm{Sb}_{2} \mathrm{Te}_{5}$. The electric field, magnetic field and current distributions in the absorber are investigated to explain the physical origin of the absorbance. Moreover, we carried out numerical simulations to investigate the temporal variation of temperature in the $\mathrm{Ge}_{2} \mathrm{Sb}_{2} \mathrm{Te}_{5}$ layer and to show that the temperature of amorphous $\mathrm{Ge}_{2} \mathrm{Sb}_{2} \mathrm{Te}_{5}$ can be raised from room temperature to > $433 \mathrm{~K}$ (amorphous-to-crystalline phase transition temperature) in just $0.37 \mathrm{~ns}$ with a low light intensity of $95 \mathrm{nW} / \mu^{2}{ }^{2}$, owing to the enhanced broadband light absorbance through strong plasmonic resonances in the absorber. The proposed phase-change metamaterial provides a simple way to realize a broadband perfect absorber in the visible and near-infrared (NIR) regions and is important for a number of applications including thermally controlled photonic devices, solar energy conversion and optical data storage.

ince the first demonstration by Landy et al. ${ }^{1}$, metamaterial (MM) absorbers have stimulated a new research field of clear relevance for solar energy harvesting application $\mathrm{s}^{2-5}$ due to their near unity absorbance, wideangle incidence and polarization independence. Besides its polarization and incident angle dependence, the bandwidth of a MM absorber also plays a crucial role in its design. To date, many efforts have been made to obtain MM perfect absorbers at microwave ${ }^{1}$, terahert $z^{6}$ and infrared frequencies ${ }^{7-9}$. As the electric and magnetic resonance frequencies of MMs are determined by their geometry, the MMs are limited to a narrow frequency region. However, a resonance response over a wide frequency range is desired for thin-film thermal emitters ${ }^{10}$, thermo photovoltaic cells ${ }^{7}$, and plasmonic scatters ${ }^{11}$.To this end, dual-band ${ }^{12-14}$, triple-band ${ }^{15,16}$ and tunable-band ${ }^{17} \mathrm{MM}$ absorbers have been demonstrated. A few recent works have been presented to directly achieve a broadband perfect $M M$ absorber, mostly at terahertz frequencies ${ }^{18-25}$, whereas broadband MM perfect absorbers in the visible regime still remain a challenge. This is because from the MMs are based on metal-dielectric-metal(MDM) multilayers, where the pitch of the MM elements is much smaller than the resonant absorption peak wavelength in order to prevent high order diffraction ${ }^{26}$ thus leading to an extremely small resonant element that is hard to realize experimentally in the visible regime. Quite recently, Aydin et al..$^{5}$ demonstrated an ultrathin plasmonic super absorber consisting of a metal-dielectric-metal film with a nanostructured top silver film composed of crossed trapezoidal arrays. Such a MM absorber has a broadband and polarization-independent resonant light absorption over the entire visible spectrum. However, the complex nanofabrication requirements of the crossed trapezoidal arrays may limit practical device applications.

MM absorbers consist of a triple-layer metal-dielectric-metal thin film laminate where only the top metal layer is patterned that serves as an electric resonator. The bottom metal layer acts as an optical mirror to eliminate the transmittance. The coupling between two metallic layers results in magnetic resonance depending on the dielectric constant of the dielectric layer ${ }^{27}$, so the dielectric material placed in between the metal layers, plays an important role in manipulating the resonance conditions for perfect absorption. Indeed absorption is greatly enhanced via critical coupling to a cavity resonance when the dielectric layer has a large imaginary part of the refractive inde $\mathrm{x}^{28}$. Therefore, the problem of the narrow band absorption of a MM absorber can be tackled if the 


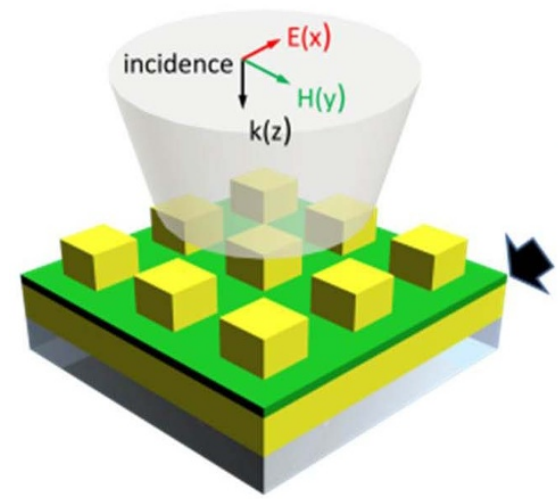

(a)

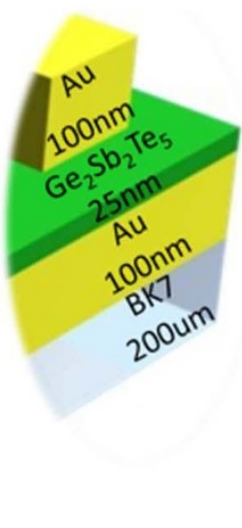

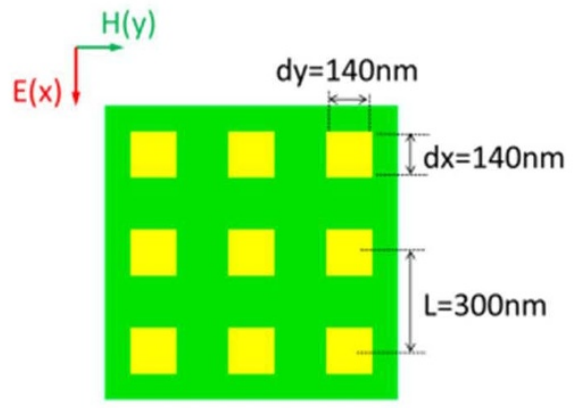

(b)

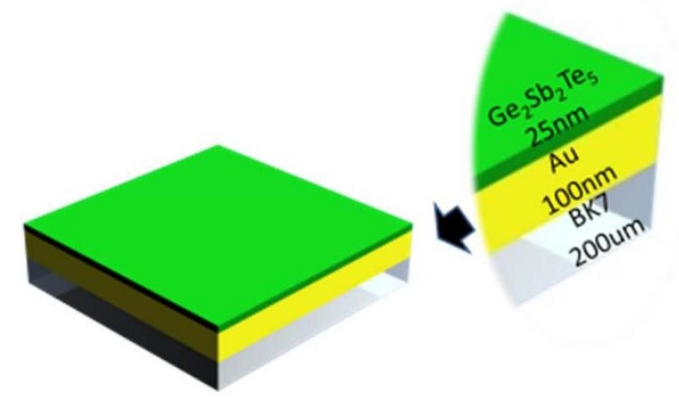

(c)

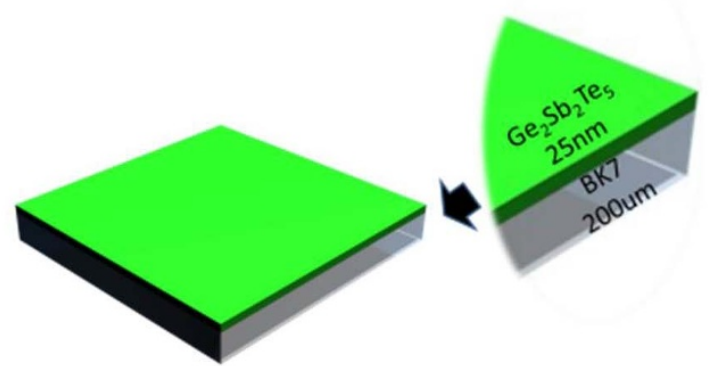

(d)

Figure $1 \mid$ (a)Schematic of the metamaterial absorber and the incident light polarization configuration. The thicknesses of $\mathrm{Au}_{\mathrm{s}} \mathrm{squares} \mathrm{Ge}_{2} \mathrm{Sb}_{2} \mathrm{Te}_{5} \mathrm{spacer}$ and Au mirror are $100 \mathrm{~nm}, 25 \mathrm{~nm}$ and $100 \mathrm{~nm}$, respectively. The lattice constant in both $x$ and $y$-directions is $L=300 \mathrm{~nm}$ and square dimension is $d_{x}=$ $d_{y}=140 \mathrm{~nm}$. The whole structure resides on a BK7 silica glass with $200 \mu \mathrm{m}$ thickness. (b) Top view of the absorber. (c) Schematic of a $25 \mathrm{~nm}$ thick $\mathrm{Ge}_{2} \mathrm{Sb}_{2} \mathrm{Te}_{5}$ dielectric layer with $100 \mathrm{~nm}$ thick ground Au layer deposited on a BK7 silica glass. (d) Schematic of the single $\mathrm{Ge}_{2} \mathrm{Sb}_{2} \mathrm{Te}_{5} \mathrm{dielectric} \mathrm{layer}$ deposited on a BK7 silica glass.

MM structural design is engineered in such a way that the middle dielectric layer has a large imaginary part of dielectric constant over a wide frequency range.

In this paper we combine a dual layer MM structure with the prototypical Phase-change material (PCM), $\mathrm{Ge}_{2} \mathrm{Sb}_{2} \mathrm{Te}_{5}$, to produce a $\mathrm{MM}$ absorber exhibiting a broadband near perfect absorbance in the visible regime. The device remains highly absorbing over a wide range of incident angles for both TE and TM polarizations. For this study the Ge-Sb-Te system was selected as the active phase change material because its dielectric properties possesses a large imaginary part over the entire visible spectrum ${ }^{29}$. Most notably, $\mathrm{Ge}_{2} \mathrm{Sb}_{2} \mathrm{Te}_{5}$ is a smart material that undergoes a thermally driven amorphous-tocrystalline phase transition above $\sim 433 \mathrm{~K}^{30,31}$, which takes the material from an amorphous state to a polycrystalline cubic state. The thermal control of the phase transition is generally achieved by either photothermal heating or electrical Joule heating ${ }^{32}$. To date, this phase transition has been explored for the development of novel electronics switches ${ }^{33}$, memories ${ }^{34}$ and tunable nanostructures ${ }^{35-37}$. However, the application of PCMs requires fast crystallization. Crystallization times on the order of 10-100 ns and ideally less than $1 \mathrm{~ns}$ are necessary for non-volatile phase-change-random-access memory (PC-RAM) to compete with the speed of volatile dynamic random access memory ${ }^{38-40}$. This has restricted the selection of PCMs and raises the question of how the crystallization speed be increased. Here, we show that the proposed broadband Metamaterial Perfect Absorber (MMPA) can be used to increase the speed that energy is delivered to the $\mathrm{Ge}_{2} \mathrm{Sb}_{2} \mathrm{Te}_{5}$ with a small optical injection power of $0.03 \mathrm{~mW}$ focused into a spot of radius $10 \mu \mathrm{m}$ corresponding to a low intensity of $95 \mathrm{nW} / \mu \mathrm{m}^{2}$.
The crystallization rate of $\mathrm{Ge}_{2} \mathrm{Sb}_{2} \mathrm{Te}_{5}$ is fast, indeed electrical Joule heating of phase change memory cells has shown crystallization in just $500 \mathrm{ps}^{41}$. However, it is difficult to deliver optical energy to $\mathrm{Ge}_{2} \mathrm{Sb}_{2} \mathrm{Te}_{5}$ on similar time scales. Optically heating films thicker than a few tens of nanometers results in a large temperature difference between the film surface, where the laser radiation is focused, and the surface that is interfaced with the substrate. If the incident laser energy is increased too quickly, the $\mathrm{Ge}_{2} \mathrm{Sb}_{2} \mathrm{Te}_{5}$ surface is ablated or damaged. In contrast, the MMPA structure can be used to generate heat inside the $\mathrm{Ge}_{2} \mathrm{Sb}_{2} \mathrm{Te}_{5}$ layer and not at the surface, thus allowing realization of optical switching at the intrinsic crystallization rate of the $\mathrm{Ge}_{2} \mathrm{Sb}_{2} \mathrm{Te}_{5}$ material. More importantly, each state of the $\mathrm{Ge}_{2} \mathrm{Sb}_{2} \mathrm{Te}_{5}$ is non-volatile and reversible. This implies that phase change MMPAs have potential applications in ultra-low power consumption optical switches that do not require a continuous energy supply to maintain the state of the switch. These two features make the PCM switch a unique category when compared with the wide variety of other optical switches that have been proposed and developed. Therefore, our method can potentially reduce the power requirements for photonic devices based on a thermal phase change, paving the way for the realization of ultrafast photothermally tunable photonic devices. This work not only shows the potential for achieving a broadband MMPA but also extends the idea of incorporating traditional data storage materials within the broadband MMPA. Moreover, the proposed MMPA is developed from previously studied photonic structures and commercial data storage devices and as such is compatible with industrially scalable fabrication techniques. 

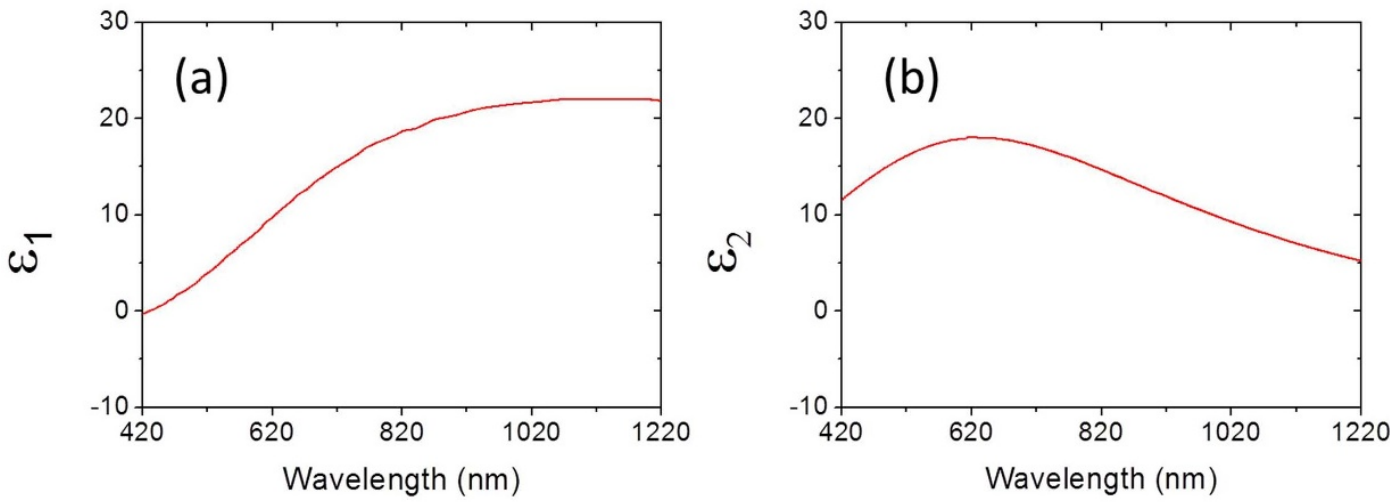

Figure $2 \mid$ Dielectric constant (a) $\varepsilon_{1}(\omega)$ vs wavelength, (b) $\varepsilon_{2}(\omega)$ vs wavelength for amorphous phase of $\mathrm{Ge}_{2} \mathrm{Sb}_{2} \mathrm{Te}_{5}$ [see reference 29].

\section{Results}

Structure and design. Our metamaterial absorber consists of two $\mathrm{Au}$ layers separated by a $\mathrm{Ge}_{2} \mathrm{Sb}_{2} \mathrm{Te}_{5}$ layer. Fig. 1(a)-(c) shows a schematic drawing of the tri-layer metamaterial absorber, where the lattice constant is $L=300 \mathrm{~nm}$, the side length of the $\mathrm{Au}$ square is $d_{x}=d_{y}=140 \mathrm{~nm}$, the thickness of the top Au layer is $100 \mathrm{~nm}$, the $\mathrm{Ge}_{2} \mathrm{Sb}_{2} \mathrm{Te}_{5}$ layer is $25 \mathrm{~nm}$ and the bottom Au layer is $100 \mathrm{~nm}$ in order to prevent any transmission through the structure $\mathrm{e}^{42}$. A single unit can be treated as an optical resonator. The unit cells are periodically arranged to form a two-dimensional square lattice in both the $x$ and $y$ directions. Furthermore, the thick Au bottom layer also interacts with the upper Au squares to give rise to a close loop of displacement current $\left(J_{D}\right)$ and localizes the electromagnetic (EM) field within the $\mathrm{Ge}_{2} \mathrm{Sb}_{2} \mathrm{Te}_{5}$ dielectric layer. The whole structure resides on a $200 \mu \mathrm{m}$ thick BK7 glass. Au is selected as the metal due to its stability and low ohmic loss. The geometry of the Au pattern and the thickness of the sandwich layers that vitally affect the dipolar resonances have been chosen to allow for both impedance matching and strong absorbance, whilst the square shaped upper $\mathrm{Au}$ layer is expected to provide polarization independent excitation of the resonances ${ }^{43}$. For comparison, a $25 \mathrm{~nm}$ thick $\mathrm{Ge}_{2} \mathrm{Sb}_{2} \mathrm{Te}_{5}$ dielectric layer with $100 \mathrm{~nm}$ thick ground Au layer and a single $25 \mathrm{~nm}$ thick $\mathrm{Ge}_{2} \mathrm{Sb}_{2} \mathrm{Te}_{5}$ dielectric layer are also shown in Fig. 1(c) and $(\mathrm{d})$ respectively. The simulation is performed by commercial software COMSOL, which is based on the Finite Element Method (FEM). The dielectric properties of Au as given by Johnson \& Christy are used ${ }^{44}$. All the structures are excited by a source with a wavelength range from 420 to $1220 \mathrm{~nm}$, propagating along the negative $z$ direction with the E field polarized in the $x$ direction as shown in Fig. 1(a). The light source has a repetition rate, $f_{r}=25 \mathrm{kHz}$ and pulse duration of $0.25 \mathrm{~ns}$. The light fluence illuminating the sample from a single pulse is written as ${ }^{45}$

$$
F_{l}(r)=\frac{2 P_{0}}{\pi w^{2} f_{r}} \exp \left(-\frac{2 r^{2}}{w^{2}}\right)
$$

where $P_{0}=0.03 \mathrm{~mW}$ is the total power of the injection light, $r$ is the distance from the beam center, $w=10 \mu \mathrm{m}$ is Gaussian beam waist. Scattering boundary conditions are applied in the $z$ direction and periodic boundaries are used for a unit cell in the $x-y$ plane. The frequency dependent reflectance $R(\omega)$ and transmittance $T(\omega)$ were abstained from the S-parameters in the simulation package and the absorbance, $A(\omega)$, was calculated as $A(\omega)=1-T(\omega)-$ $R(\omega)^{9}$.

Optical properties of $\mathbf{G e}_{2} \mathbf{S b}_{\mathbf{2}} \mathbf{T} \mathbf{e}_{5}$. The real, $\varepsilon_{1}(\omega)$ and imaginary, $\varepsilon_{2}(\omega)$ parts of the dielectric function of $\mathrm{Ge}_{2} \mathrm{Sb}_{2} \mathrm{Te}_{5}$ in the amorphous phase are obtained from the experimental data in Ref. 29 and for the entire visible and near-infrared (NIR) spectral ranges the dielectric function is shown in Fig. 2. As can be seen, the dielectric constant of $\mathrm{Ge}_{2} \mathrm{Sb}_{2} \mathrm{Te}_{5}$ is very dispersive and has a large imaginary part indicating a high absorption coefficient. Thereby replacing the dielectric spacer with $\mathrm{Ge}_{2} \mathrm{Sb}_{2} \mathrm{Te}_{5}$ in MDM-MM absorber could enable broadband absorption.

Simulated optical properties of the broadband metamaterial perfect absorber. Firstly, we optimize the MMPA at normally incident TE (or TM) plane waves as shown in Fig. 1. The simulated reflectance $R(\omega)=|r|^{2}$ and absorbance $A(\omega)$ of the MMPA, an amorphous $\mathrm{Ge}_{2} \mathrm{Sb}_{2} \mathrm{Te}_{5}$ dielectric layer with ground Au layer, and monolithic $\mathrm{Ge}_{2} \mathrm{Sb}_{2} \mathrm{Te}_{5}$ dielectric layer at normal incidence are presented in Fig. 3, where $r$ is the complex reflection coefficient. As can be seen, the MMPA possesses a low reflectance owing to impedance matching to the vacuum, leading to a strong absorbance over the entire visible region. However, the single amorphous $\mathrm{Ge}_{2} \mathrm{Sb}_{2} \mathrm{Te}_{5}$
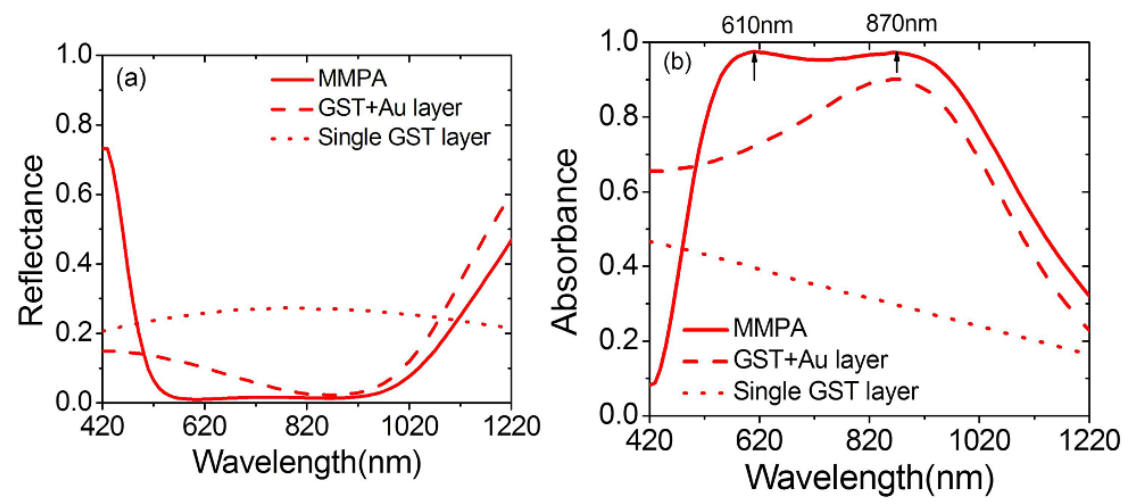

Figure 3 |3D- FEM simulation of spectrum of (a) reflectance, (b) absorbance for the MMPA, $\mathrm{Ge}_{2} \mathrm{Sb}_{2} \mathrm{Te}_{5}$ dielectric layer with ground Au layer, and single $\mathrm{Ge}_{2} \mathrm{Sb}_{2} \mathrm{Te}_{5}$ dielectric layer at normal incidence. 


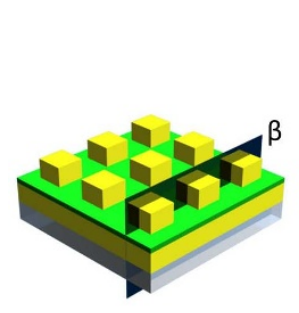

(a)

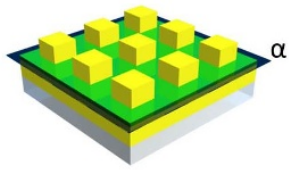

(b)

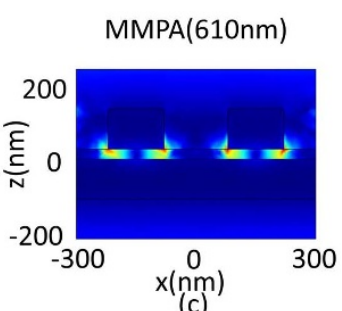

(c)

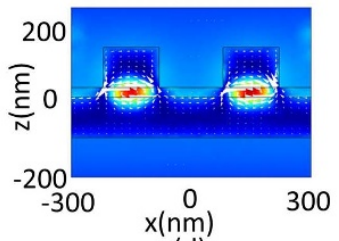

(d)

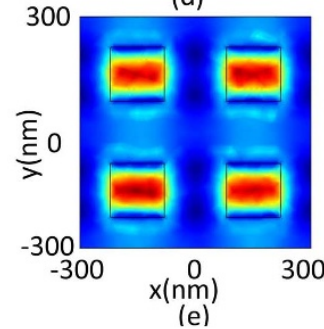

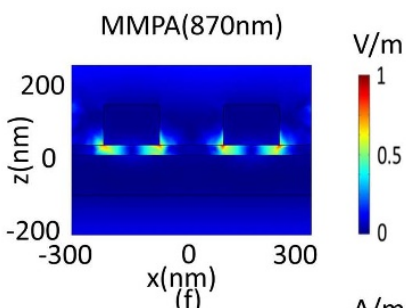

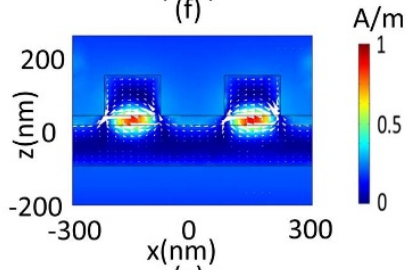

(g)

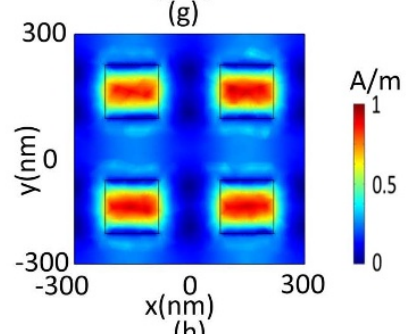

(h)

Figure $4 \mid$ (a) Three dimensional schematic view of the MMPA showing the cross section plane $\beta$ along $x-z$ plane. (b) Three dimensional schematic view of the MMPA showing the cross section plane $\alpha$ along $x-y$ plane at the top Au/bottom $\mathrm{Ge}_{2} \mathrm{Sb}_{2} \mathrm{Te}_{5}$ interface. (c) Total electric field intensity distribution along the $\beta$ plane for the MMPA at $\lambda_{1}=610 \mathrm{~nm}$. (d) The distributions of total magnetic field intensity distribution and displacement current $\left(J_{D}\right)$ along the $\beta$ plane for the MMPA at $\lambda_{1}=610 \mathrm{~nm}$. (e) Total magnetic field intensity distribution in $\alpha$ plane for the MMPA at $\lambda_{1}=610 \mathrm{~nm}$. (f) Total electric field intensity distribution along the $\beta$ plane for the MMPA at $\lambda_{2}=870 \mathrm{~nm}$. (g) The distributions of total magnetic field intensity distribution and displacement current $\left(J_{D}\right)$ along the $\beta$ plane for the MMPA at $\lambda_{2}=870 \mathrm{~nm}$. (h) Total magnetic field intensity distribution in $\alpha$ plane for the MMPA at $\lambda_{2}=870 \mathrm{~nm}$.
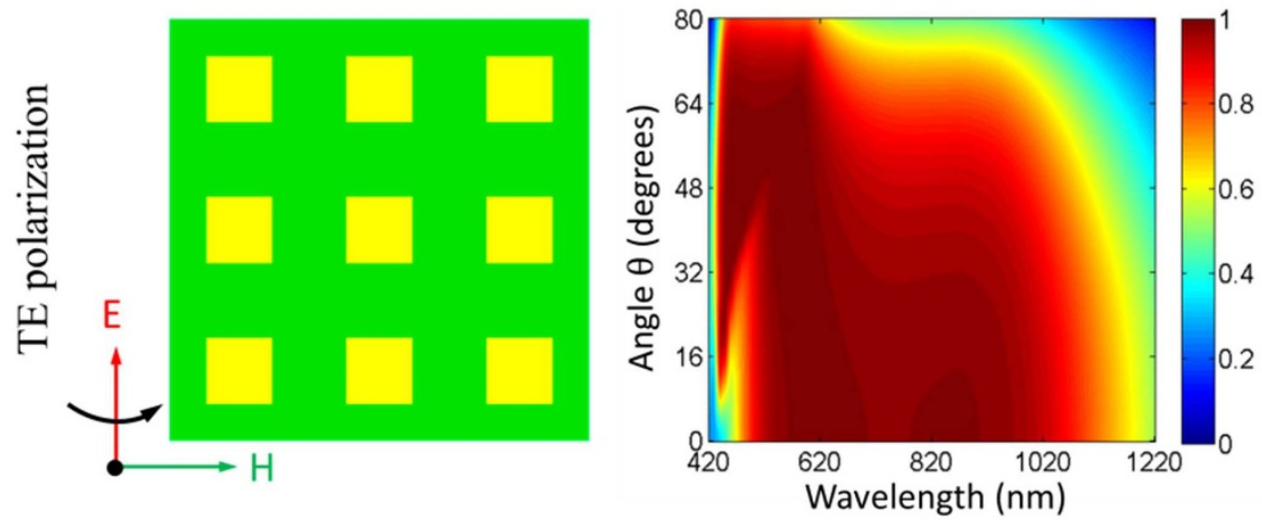

(a)
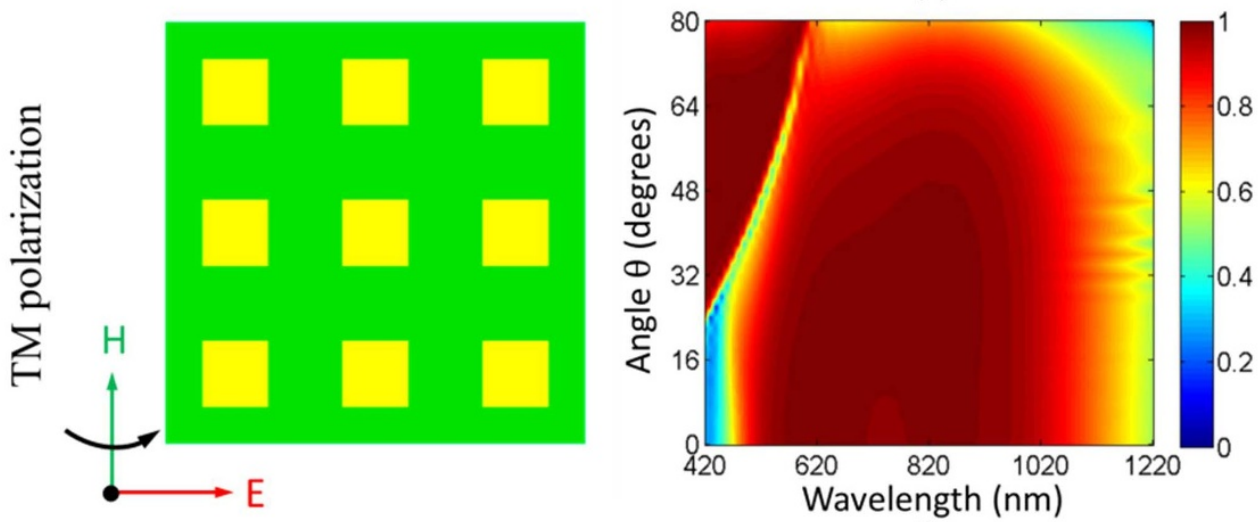

(b)

Figure 5 3D- FEM simulation of angular dispersions of the absorbance peaks (a) for amorphous $\mathrm{Ge}_{2} \mathrm{Sb}_{2} \mathrm{Te}_{5}$ at $\mathrm{TE}$ polarization, (b) for amorphous $\mathrm{Ge}_{2} \mathrm{Sb}_{2} \mathrm{Te}_{5}$ at $\mathrm{TM}$ polarization. 
TM

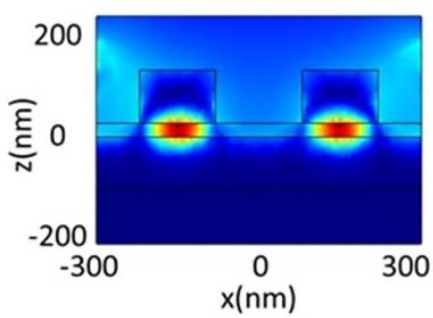

(a) $\theta=0^{\circ}$

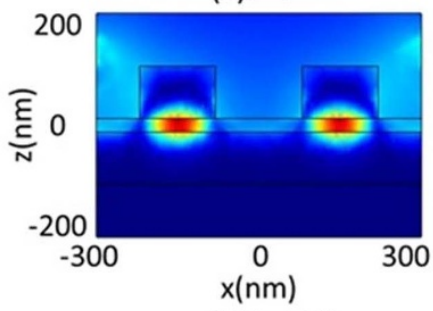

(b) $\theta=30^{\circ}$

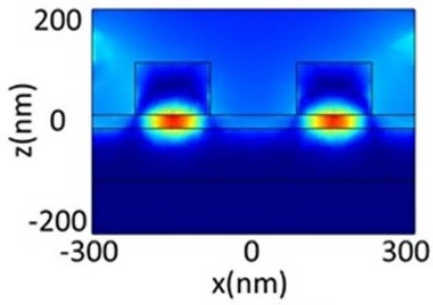

(c) $\theta=60^{\circ}$

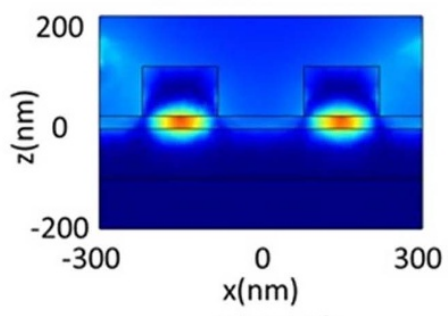

(d) $\theta=80^{\circ}$
TE

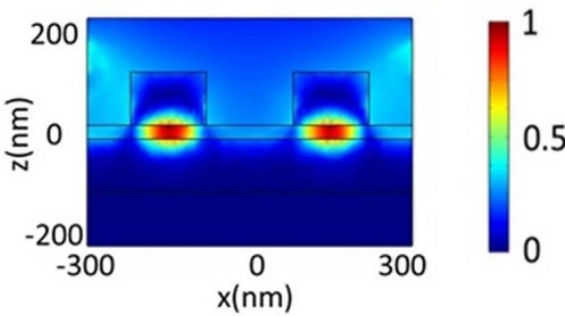

(e) $\theta=0^{\circ}$
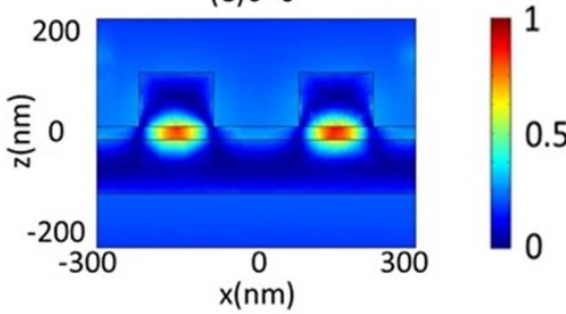

(f) $\theta=30^{\circ}$

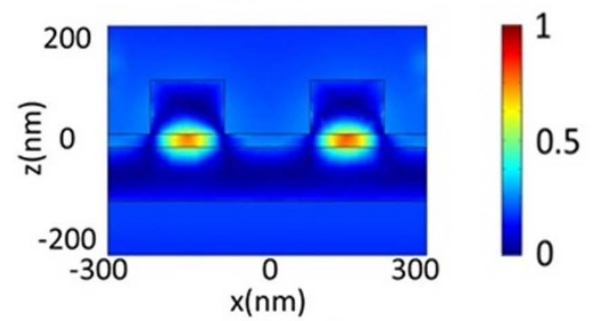

(g) $\theta=60^{\circ}$

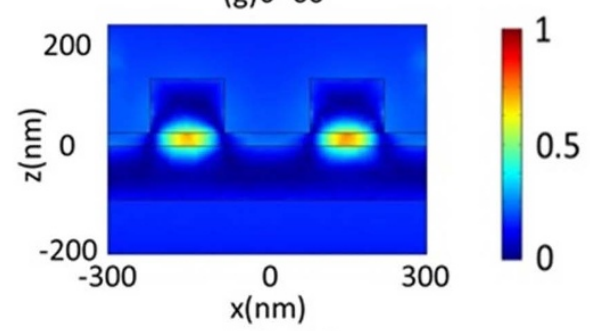

(h) $\theta=80^{\circ}$

Figure 6 | 3D-FEM simulation of total magnetic field distribution for TM polarization at (a) $\theta=0^{\circ}$, (b) $\theta=30^{\circ}$, (c) $\theta=60^{\circ},(\mathrm{d}) \theta=80^{\circ}$; For TE polarization at (e) $\theta=0^{\circ}$, (f) $\theta=30^{\circ}$, (g) $\theta=60^{\circ}$, (h) $\theta=80^{\circ}$ where $\lambda_{1}=610 \mathrm{~nm}$.

film possesses the highest reflectance and lowest absorbance among the different structures due to the absence of the metal layers. In the MMPA, it is clear that a wide band near-perfect absorption around $670 \mathrm{~nm}$ is obtained with the absorbance of $96.8 \%$ at $\lambda_{1}=610 \mathrm{~nm}$ and $96.2 \%$ at $\lambda_{2}=870 \mathrm{~nm}$. These two resonances are expected to account for this wide band near-perfect absorbance ${ }^{7,46}$.

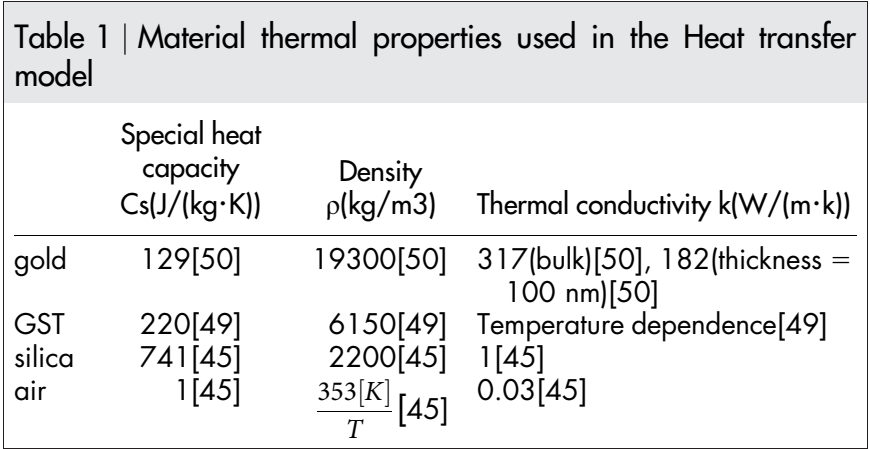

As stated previously, the top Au square array is responsible for electric dipolar resonances while the back Au layer is mainly used for creating magnetic dipolar resonances between the two metallic layers. Therefore, to gain insight on the localized surface plasmon resonance and the effect of $\mathrm{Ge}_{2} \mathrm{Sb}_{2} \mathrm{Te}_{5}$ in broadening the absorbance spectrum, we calculate the electromagnetic near-field distribution at normal incidence. Fig. 4 shows the field distributions and $J_{D}$ for the maximum absorbance of 0.968 at $\lambda_{1}=610 \mathrm{~nm}$ and 0.962 at $\lambda_{2}=$ $870 \mathrm{~nm}$ for the MMPA. In the pattern, the white arrows represent $J_{D}$ whereas the color represents the magnitude of the electromagnetic field intensities.

The total electric field intensity distribution associated with $\mathrm{A}(\omega)$ $=0.968$ at $\lambda_{1}=610 \mathrm{~nm}$ for the amorphous $\mathrm{Ge}_{2} \mathrm{Sb}_{2} \mathrm{Te}_{5}$ along a crosssection plane, $\beta$ (as indicated in Fig. 4(a)) is shown in Fig. 4(c). It clearly shows that the electric field is mostly concentrated in the amorphous $\mathrm{Ge}_{2} \mathrm{Sb}_{2} \mathrm{Te}_{5}$ layer to excite the electric resonance moments. In Fig. 4(d), it shows that the total magnetic field intensity, along the $\beta$ plane is also strongly localized in the amorphous $\mathrm{Ge}_{2} \mathrm{Sb}_{2} \mathrm{Te}_{5}$ dielectric layer. This is caused by the anti-parallel $J_{D}$ (shown as the white arrows in Fig. 4(d)) creating a loop between the Au square rods array and the bottom $\mathrm{Au}$ film ${ }^{17}$. These loops in 

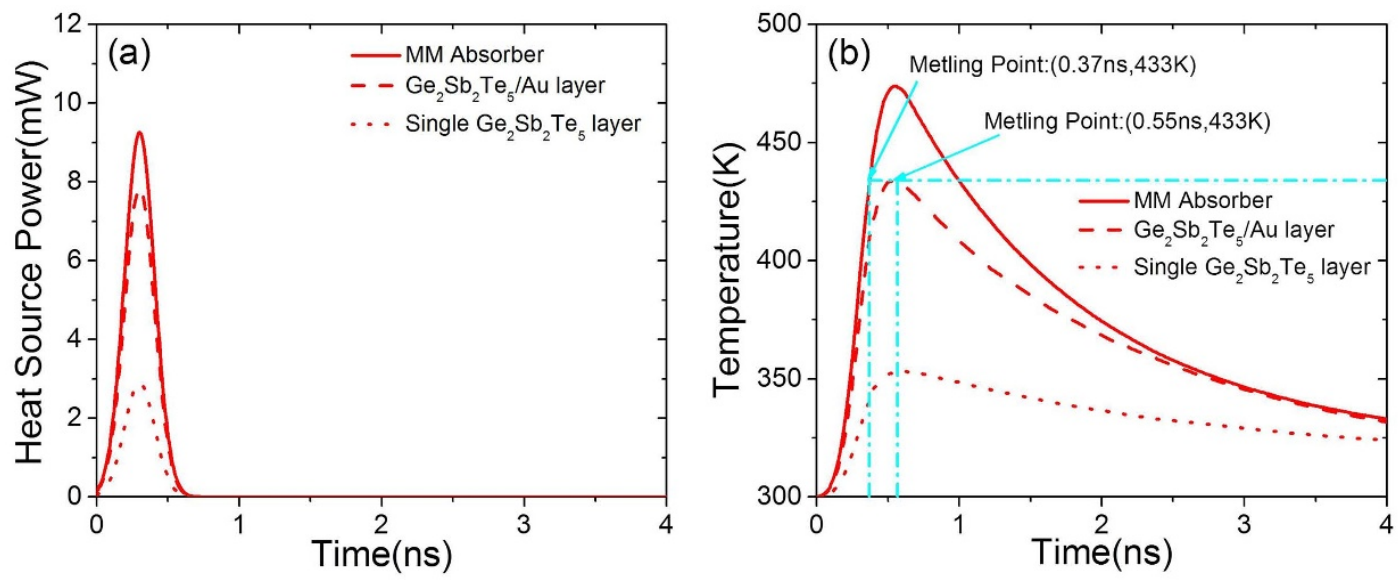

Figure $7 \mid$ (a) 3D-FEM simulation of heat power irradiating on the MMPA (solid line), a $25 \mathrm{~nm}$ thick amorphous $\mathrm{Ge}_{2} \mathrm{Sb}_{2} \mathrm{Te}_{5}$ dielectric layer with $100 \mathrm{~nm}$ thick ground Au layer (dashed line), and $25 \mathrm{~nm}$ thick monolithic amorphous $\mathrm{Ge}_{2} \mathrm{Sb}_{2} \mathrm{Te}_{5}$ layer located at the beam center (dotted line), (b) Temperature during one pulse of amorphous $\mathrm{Ge}_{2} \mathrm{Sb}_{2} \mathrm{Te}_{5}$ layer in the MMPA (solid line), $\mathrm{Ge}_{2} \mathrm{Sb}_{2} \mathrm{Te}_{5}$ dielectric layer with ground Au layer (dashed line), single $\mathrm{Ge}_{2} \mathrm{Sb}_{2} \mathrm{Te}_{5}$ dielectric layer (dotted line).

$J_{D}$ give rise to artificial magnetic dipole moments that interact strongly with the incident magnetic field. Therefore, our structure provides a simultaneous excitation of electric and magnetic resonances to enhance the localized electromagnetic fields ${ }^{47}$. In Fig. 4(e), we depict the $\mathrm{H}$ field intensity in the interface plane of the top Au layer and $\mathrm{Ge}_{2} \mathrm{Sb}_{2} \mathrm{Te}_{5}$ dielectric layer, shown as the $\alpha$ plane in Fig. 4(b). It can be seen that the $\mathrm{H}$ field intensity is stronger in the centre of the unit cell indicating the strong magnetic dipolar moments formed between the Au square rods array and the bottom Au film 5 . In Figs. 4(f)-4(h), we show the total electric field, magnetic field, and $\mathrm{J}_{\mathrm{D}}$, respectively, associated with $\mathrm{A}(\omega)=0.962$ at $\lambda_{2}=$ $870 \mathrm{~nm}$. As can be seen, the field maps of the structure are similar with the ones at $\lambda_{1}=610 \mathrm{~nm}$, which implies that the electrical resonant dipole and magnetic resonant dipole can also be simultaneously excited to create a strong absorbance.

Ideally, the absorbance of the perfect absorber should be maintained at a large incidence angle and not depend on the light polarization ${ }^{5}$. Thus, Fig. 5 presents the absorbance as a function of wavelength and angle of incidence, where (a)-(b) show the absorbance for TE and TM polarization in the MMPA with an amorphous $\mathrm{Ge}_{2} \mathrm{Sb}_{2} \mathrm{Te}_{5}$ dielectric layer and the incident angle $\left(\theta_{i}\right)$ is varied in $1^{\circ}$ steps from $0^{\circ}$ to $80^{\circ}$. A broadband absorbance remains around 0.45 even at incident angle of $80^{\circ}$ for TE polarization and 0.55 at $80^{\circ}$ for TM polarization over the entire visible and NIR regimes. As can be seen, the increased $\theta_{i}$ results in an increment of absorbance below $450 \mathrm{~nm}$, which is around the plasma frequency of bulk Au. With the higher angles of incidence, the path length of light in the nanostructures becomes longer, thus leading to a stronger absorbance of the light ${ }^{5}$. However, at longer wavelength, increasing $\theta_{i}$ leads to decreased absorbance. This is because the magnetic fields at large incident angles are confined less efficiently in the $\mathrm{Ge}_{2} \mathrm{Sb}_{2} \mathrm{Te}_{5}$ dielectric layer than at normal incidence, hence leading to a weaker magnetic dipolar resonance ${ }^{9}$ as shown in Fig. 6. In Fig. 5, note that there is a good spectral overlap between the absorbance for TE and TM polarizations. Thus, the proposed broadband MMPA is polarization-independent over a wide range of incident angles across the visible and NIR frequency regions.

Further simulations were performed to study the relationship between the absorbance, the strength of the magnetic resonance at different oblique angles of the incidence. In Fig. 6, the total magnetic field intensity distributions at $\lambda_{1}=610 \mathrm{~nm}$ are investigated. It can be seen that the absorber maintains the localized magnetic field intensity thus the orientation of the magnetic resonant dipole at large

Time $=0.37 n s$, Slice $=$ Temperature $[K]$,Arrow:Total Heat Flux
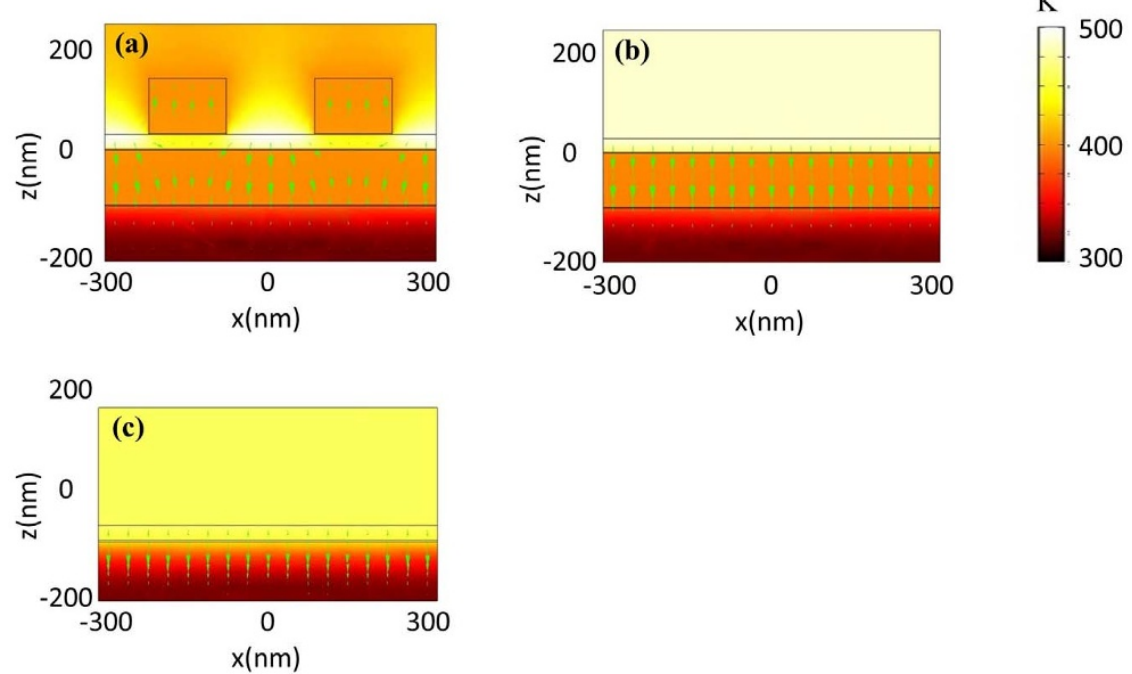

Figure $8 \mid$ The cross section view of the unit cell of the (a) MMPA, (b) $\mathrm{Ge}_{2} \mathrm{Sb}_{2} \mathrm{Te}_{5}$ layer with Au layer, (c) Monolithic $\mathrm{Ge}_{2} \mathrm{Sb}_{2} \mathrm{Te}_{5}$ layer, where the color indicates the temperature distribution and the arrows indicate the heat flux at $0.37 \mathrm{~ns}$. 
incident angles. In particular, the localized magnetic field intensity for TM polarization shown in Fig. 6(a)-(d) is confined more strongly than for TE polarization shown in Fig. 6(e)-(h) at different incident angles. This is because the direction of the magnetic field of TM polarized light does not change significantly with incident angles and it can efficiently drive the current loop at all incident angles. Conversely, for the TE polarization, the magnetic field drives the circulating currents less efficiently at large angles ${ }^{48}$.

Fast phase transition of the $\mathrm{Ge}_{2} \mathrm{Sb}_{2} \mathrm{Te}_{5}$ in the metamaterial perfect absorber. The absorption of electromagnetic radiation in MMPA is mainly due to losses in the dielectric interlayer. Therefore $\mathrm{Ge}_{2} \mathrm{Sb}_{2} \mathrm{Te}_{5}$ is very promising and gives rise to a high and flat broadband absorbance due to its large imaginary part of the dielectric constant over the entire visible range. This strong absorbance allows rapid heating of the $\mathrm{Ge}_{2} \mathrm{Sb}_{2} \mathrm{Te}_{5}$ at visible wavelengths thus leading to a fast phase transition of the $\mathrm{Ge}_{2} \mathrm{Sb}_{2} \mathrm{Te}_{5}$ layer from the amorphous to crystalline state. To show this, a heat transfer model is used to investigate the temporal variation of temperature of $\mathrm{Ge}_{2} \mathrm{Sb}_{2} \mathrm{Te}_{5}$ layer for different structures using the Finite Element Method (FEM) solver within COMSOL. The material thermal properties used for the simulation are summarized in Table 1. The change in thermal conductivity of $\mathrm{Ge}_{2} \mathrm{Sb}_{2} \mathrm{Te}_{5}$ with the temperature is obtained from experimental data in Ref. 49. In this simulation, the thermal energy absorbed by one unit cell is defined as Ref. 45

$$
E_{t h}(r)=R_{a} \times L^{2} \times F_{l}(r)
$$

where $L=300 \mathrm{~nm}$ is the lattice constant of the metamaterials, $R_{a}$ is 0.8952 for the MMPA, 0.7582 for the $25 \mathrm{~nm}$ thick $\mathrm{Ge}_{2} \mathrm{Sb}_{2} \mathrm{Te}_{5}$ dielectric layer with $100 \mathrm{~nm}$ thick ground Au layer, and 0.2797 for the monolithic $25 \mathrm{~nm}$ thick $\mathrm{Ge}_{2} \mathrm{Sb}_{2} \mathrm{Te}_{5}$ dielectric layer respectively. $R_{a}$ is derived from the overlap integral between the light source power density spectrum and the absorbance spectrums of different structures, shown in Fig. 3(b), in the wavelength range from $420 \mathrm{~nm}$ to $1220 \mathrm{~nm}$. The heat source power for different structures is then described by

$$
Q_{s}(r, t)=E_{t h}(r) \frac{1}{\sqrt{\pi} r} \exp \left(-\frac{\left(t-t_{0}\right)^{2}}{\tau^{2}}\right)
$$

where $\tau=0.15 \mathrm{~ns}$ is the time constant of the light pulse, $t_{0}=0.3 \mathrm{~ns}$ is the time delay of the pulse peak. Fig. 7(a) shows the heat source power: $Q_{s}(r, t)$ for the different structures, where the structures are located at the center of a Gaussian beam, and Fig. 7(b) shows the comparison of temperatures in $\mathrm{Ge}_{2} \mathrm{Sb}_{2} \mathrm{Te}_{5}$ dielectric layer for the three structures.

In Fig. 7(b), the numerical simulation shows that the temperature within the amorphous $\mathrm{Ge}_{2} \mathrm{Sb}_{2} \mathrm{Te}_{5}$ dielectric layer of the MMPA is a function of the incident radiation flux and can exceed the amorphous to crystalline phase transition temperature of $433 \mathrm{~K}$ after $0.37 \mathrm{~ns}$ and a maximum of $474 \mathrm{~K}$ after $0.56 \mathrm{~ns}$ for a threshold incident flux of $95 \mathrm{nW} / \mu \mathrm{m}^{2}$. Due to heat dissipation and radiation to the surroundings, the MMPA begins to cool after $0.56 \mathrm{~ns}$ before the next pulse arrives. Eventually the temperature falls below the cubic crystal transition temperature. We have verified that a $\mathrm{Ge}_{2} \mathrm{Sb}_{2} \mathrm{Te}_{5}$ dielectric layer with ground $\mathrm{Au}$ layer can also reach the cubic crystallisation temperature of $433 \mathrm{~K}$, but the time is $0.55 \mathrm{~ns}$, nearly $0.18 \mathrm{~ns}$ longer than the MMPA. However, a bare film of $\mathrm{Ge}_{2} \mathrm{Sb}_{2} \mathrm{Te}_{5}$ cannot obtain $433 \mathrm{~K}$ under the same incident flux of $95 \mathrm{nW} / \mu \mathrm{m}^{2}$. A large temperature difference of $122 \mathrm{~K}$ at $0.56 \mathrm{~ns}$ between the MMPA and monolithic $\mathrm{Ge}_{2} \mathrm{Sb}_{2} \mathrm{Te}_{5}$ layer is clearly shown in Fig. 7(b); thus showing the usefulness of a metamaterial absorber for increasing the rate at which heat is delivered to $\mathrm{Ge}_{2} \mathrm{Sb}_{2} \mathrm{Te}_{5}$. Here, we do not consider the change in the optical properties during the phase transition of $\mathrm{Ge}_{2} \mathrm{Sb}_{2} \mathrm{Te}_{5}$. Here, we focus on showing that the MMPA decreases the time necessary to reach the amorphous to polycrystalline cubic phase transition temperature.
The temperature distribution of the structures at $0.37 \mathrm{~ns}$ is shown in Fig. 8(a), (b) and (c) respectively. One can observe that the temperature within amorphous $\mathrm{Ge}_{2} \mathrm{Sb}_{2} \mathrm{Te}_{5}$ dielectric layer of the MMPA is the highest. In all of the structures, the dominant temperature gradient is along the same direction as the incident light, indicating that BK7 silica substrate is an effective heat $\sin k^{45}$.

\section{Discussion}

In conclusion, we have presented a simple design of a MMPA that shows a polarization-independent near perfect broadband absorbance in the visible frequency regions due to the inclusion of a thin film of phase change material $\left(\mathrm{Ge}_{2} \mathrm{Sb}_{2} \mathrm{Te}_{5}\right)$ that exhibits a large imaginary part of its dielectric constant. Total $\mathrm{E}$ and $\mathrm{H}$ intensity distributions have been presented to show that the electromagnetic field can be trapped efficiently inside the absorber over a wide range of incident angles. Furthermore, the absorbance spectra show substantial overlap between TE and TM polarizations. We have also studied the heat generation and temperature distribution within the structures, which triggers the amorphous to crystalline phase transition in $\mathrm{Ge}_{2} \mathrm{Sb}_{2} \mathrm{Te}_{5}$ for a low light intensity of $95 \mathrm{nW} / \mu \mathrm{m}^{2}$. In contrast, the time required to reach the amorphous to crystal phase transition temperature of the $\mathrm{Ge}_{2} \mathrm{Sb}_{2} \mathrm{Te}_{5}$ layer with a $\mathrm{Au}$ ground layer (0.55 ns) are longer than the $\mathrm{Ge}_{2} \mathrm{Sb}_{2} \mathrm{Te}_{5}$ layer in MMPA (0.37 ns) under the same conditions of optical illumination. Therefore, such a MMPA can be applied to achieve fast and energy efficient optical devices based on PCMs, and can find numerous applications in energy harvesting, biology and optical storage.

\section{Methods}

For the numerical calculations, we used the Finite Element Method by means of COMSOL Multiphysics. The unit cell of the phase-change metamaterial is shown in Fig. 1. The incident light is normal to the $\mathrm{x}-\mathrm{y}$ plane with $\mathrm{E}$ field polarized in $x$ direction. To account for the periodic nature of the metamaterial, the model boundary at $x= \pm \frac{L}{2}$ and $y= \pm \frac{L}{2}$ is set to condition of perfect electric conductor and perfect magnetic conductor, respectively for normal incidence and Floquet boundary condition for oblique incidence. Scattering boundary conditions are applied in the $z$ direction. The optical constant of gold is taken from the data of Johnson and Christy ${ }^{44}$.The dielectric function of $\mathrm{Ge}_{2} \mathrm{Sb}_{2} \mathrm{Te}_{5}$ in different phases are obtained from the experimental data $\mathrm{in}^{29}$.

1. Landy, N. I. et al. Perfect metamaterial absorber. Phys. Rev. Lett. 100, 207402 (2008).

2. Atwater, H. A. \& Polman, A. Plasmonics for improved photovoltaic devices. Nat. Mater. 9, 205-214 (2010).

3. Nagpal, P., Lindquist, N. C., Oh, S. H. \& Norris, D. J. Ultra-Smooth Patterned Metals for Plasmonics and Metamaterials. Science 325, 594-597 (2009).

4. Halas, N. J. et al. Plasmons in Strongly Coupled Nanostructures. Chem. Rev. 111, 3913-3961 (2011).

5. Aydin, K., Ferry, V. E., Briggs, R. M. \& Atwater, H. A. Broadband polarizationindependent resonant light absorption using ultrathin plasmonic super absorbers. Nat. Commun. 2, 517 (2011).

6. Tao, H. et al. A metamaterial absorber for the terahertz regime: design, fabrication and characterization. Opt. Express 16, 7181-7188 (2008).

7. Avitzour, Y., Urzhumov, Y. A. \& Shvets, G. Wide-angle infrared absorber based on a negative-index plasmonic metamaterial. Phys. Rev. B 79, 045131 (2009).

8. Liu, X., Starr, T., Starr, A. F. \& Padilla, W. J. Infrared spatial and frequency selective metamaterial with near-unity absorbance. Phys. Rev. Lett. 104, 207403 (2010).

9. Liu, N. et al. Infrared perfect absorber and its application as plasmonic sensor. Nano Lett. 10, 2342-2348 (2010).

10. Greffet, J. J. et al. Coherent emission of light by thermal sources. Nature 416, 61-64 (2002).

11. Pala, R. A. et al. Design of plasmonic thin-film solar cells with broadband absorption enhancements. Adv. Mater. 21, 3504-3509 (2009).

12. Wen, Q. Y. et al. Dual band terahertz metamaterial absorber: Design, fabrication, and characterization. Appl. Phys. Lett. 95, 241111 (2009).

13. Tao, H. et al. A dual band terahertz metamaterial absorber. J. Phys. D 43, 225102 (2010).

14. Ma, Y. et al. A terahertz polarization insensitive dual band metamaterial absorber. Opt. Lett. 36, 945-947 (2011).

15. Li, H. et al. Ultrathin multiband gigahertz metamaterial absorbers. J. Appl. Phys. 110, 014909 (2011) 
16. Shen, X. et al. Polarization-independent wide-angle triple-band metamaterial absorber. Opt. Express 19, 9401 (2011).

17. Cao, T., Zhang, L., Simpson, R. E. \& Cryan, M. J. Mid-infrared tunable polarization-independent perfect absorber using a phase-change metamaterial J.Opt.Soc.Am.B 30, 1580-1585 (2013).

18. Hu, C. et al. Mixed plasmons coupling for expanding the bandwidth of nearperfect absorption at visible frequencies. Opt. Express 17, 16745-16749 (2009).

19. Ye, Y. Q., Jin, Y. \& He, S. Omnidirectional, polarization-insensitive and broadband thin absorber in the terahertz regime. J. Opt. Soc. Am. B 27, 498-504 (2010).

20. Wakatsuchi, H., Greedy, S., Christopoulos, C. \& Paul, J. Customised broadband metamaterial absorbers for arbitrary polarization. Opt. Express 18, 22187-22198 (2010).

21. Luo, H., Cheng, Y. Z. \& Gong, R. Z. Numerical study of metamaterial absorber and extending absorbance bandwidth based on multi-square patches. Eur. Phys. J. B 81, 387-392 (2011)

22. Alici, K. B., Turhan, A. B., Soukoulis, C. M. \& Ozbay, E. Optically thin composite resonant absorber at the near-infrared band: a polarization independent and spectrally broadband configuration. Opt. Express 19, 14260 (2011).

23. Grant, J. et al. Polarization insensitive, broadband terahertz metamaterial absorber. Opt. Lett. 36, 3476-3478 (2011).

24. Liu, X. et al. Taming the Blackbody with Infrared Metamaterials as Selective Thermal Emitters. Phys. Rev. Lett. 107, 045901 (2011).

25. Huang, L. et al. Experimental demonstration of terahertz metamaterial absorbers with a broad and flat high absorption band. Opt. Lett. 37, 154-156 (2012).

26. Lee, H. M. \& Wu, J. C. Temperature Controlled Perfect Absorber Based on MetalSuperconductor-Metal Square Array. IEEE Trans. Magn. 48, 4243-4246 (2012).

27. Hao, J. et al. High performance optical absorber based on a plasmonic metamaterial. Appl. Phys. Lett. 96, 251104 (2010).

28. Kats, M. A. et al. Ultra-thin perfect absorber employing a tunable phase change material. Appl. Phys. Lett. 101, 221101 (2012).

29. Shportko, K. et al. Resonant bonding in crystalline phase-change materials. Nature 7, 653-658 (2008).

30. Michel, A. U. et al. Using Low-Loss Phase-Change Materials for Mid-Infrared Antenna Resonance Tuning. Nano. Lett. 13, 3470-3475 (2013).

31. Orava, J. et al. Optical properties and phase change transition in $\mathrm{Ge}_{2} \mathrm{Sb}_{2} \mathrm{Te}_{5}$ flash evaporated thin films studied by temperature dependent spectroscopic ellipsometry. J.Appl.Phys. 104, 043523 (2008).

32. Orava, J. et al. Characterization of supercooled liquid $\mathrm{Ge}_{2} \mathrm{Sb}_{2} \mathrm{Te}_{5}$ and its crystallization by ultra-fast-heating calorimetry. Nat. Mater. 11, 279-283 (2012).

33. Samson, Z. L. et al. Metamaterial electro-optic switch of nanoscale thickness. Appl. Phys. Lett. 96, 143105 (2010).

34. Yang, Z., Ko, C. \& Ramanathan, S. Oxide Electronics Utilizing Ultrafast MetalInsulator Transitions. Annu. Rev. Mater. Res. 41, 337-367 (2011).

35. Kats, M. A. et al. Thermal tuning of mid-infrared plasmonic antenna arrays using a phase change material. Opt. Lett. 38, 368-370 (2013).

36. Dayal, G. \& Ramakrishna, S. A. Metamaterial saturable absorber mirror. Opt. Lett. 38, 272-274 (2013).

37. Cao, T., Simpson, R. E. \& Cryan, M. J. Study of tunable negative index metamaterials based on phase-change materials. J. Opt. Soc. Am. B 30, 439-444 (2013).

38. Wuttig, M. \& Salinga, M. Phase Change Materials: Fast transformers. Nat. Mater. 11, 270-271 (2012).

39. Wang, W. J. et al. Fast phase transitions induced by picosecond electrical pulses on phase change memory cells. Appl. Phys. Lett. 93, 043121 (2008).
40. Bruns, G. et al. Nanosecond switching in GeTe phase change memory cells. Appl. Phys. Lett. 95, 043108 (2009).

41. Loke, D. et al. Breaking the Speed Limits of Phase-Change Memory. Science 336, 1566 (2012).

42. Zhang, B. et al. Polarization-independent dual-band infrared perfect absorber based on a metal-dielectric-metal elliptical nanodisk array. Opt. Express 19 15221-15228 (2011).

43. Dayal, G. \& Ramakrishna, S. A. Design of highly absorbing metamaterials for Infrared frequencies. Opt. Express 20, 17503-17508 (2012).

44. Johnson, P. B. \& Christy, R. W. Optical constants of the noble metals. Phys. Rev. B 6, 4370-4379 (1972).

45. Chen, X., Chen, Y., Yan, M. \& Qiu, M. Nanosecond Photothermal Effects in Plasmonic Nanostructures. ACS Nano 6, 2550-2557 (2012).

46. Wang, J. et al. Tunable broad-band perfect absorber by exciting of multiple plasmon resonances at optical frequency. Opt. Express 20, 14871-14878 (2012).

47. Lee, H. M. \& Wu, J. C. A wide-angle dual-band infrared perfect absorber based on metal-dielectric-metal split square-ring and square array. J. Phys. D: Appl. Phys. 45, 205101 (2012).

48. Tao, H. et al. Highly flexible wide angle of incidence terahertz metamaterial absorber: Design, fabrication, and characterization. Phys. Rev. B 78, 241103 (2008).

49. Kuwahara, M. et al. Measurement of the thermal conductivity of nanometer scale thin films by thermoreflectance phenomenon. Microelectron. Eng. 84, 1792-1796 (2007).

50. Chen, G. \& Hui, P. Thermal conductivities of evaporated gold films on silicon and glass. Appl. Phys. Lett. 74, 2942 (1999).

\section{Acknowledgments}

We acknowledge the financial support from National Natural Science Foundation of China (Grant No. 61172059, 51302026), Ph.D Programs Foundation of Ministry of Education of China (Grant No. 20110041120015), Postdoctoral Gathering Project of Liaoning Province (Grant No. 2011921008), and The Fundamental Research for the Central University(Grant No. DUT12JB01)

\section{Author contributions}

T.C., M.J.C. and R.E.S. conceived the idea of using phase-changed material for decreasing the time necessary to reach the amorphous to cubic transition temperature of $\mathrm{Ge}_{2} \mathrm{Sb}_{2} \mathrm{Te}_{5}$. T.C. designed the metamaterial and performed numerical analysis. C.W.W. and L.Z. carried out the simulations and prepared the figures. T.C. supervised the research. T.C. and M.J.C. co-wrote the manuscript

\section{Additional information}

Competing financial interests: The authors declare no competing financial interests. How to cite this article: Cao, T., Wei, C.-w., Simpson, R.E., Zhang, L. \& Cryan, M.J. Broadband Polarization-Independent Perfect Absorber Using a Phase-Change Metamaterial at Visible Frequencies. Sci. Rep. 4, 3955; DOI:10.1038/srep03955 (2014).

(c) (i) $(-)$ This work is licensed under a Creative Commons AttributionBY NC ND NonCommercial-NoDerivs 3.0 Unported license. To view a copy of this license, visit http://creativecommons.org/licenses/by-nc-nd/3.0 